\title{
Analysis of Safety Distance and Maximum Permissible Power of Resonant Wireless Power Transfer Systems with Regard to Magnetic Field Exposure
}

\author{
Young-Min Park and Jin-Kyu Byun* \\ Department of Electrical Engineering, Soongsil University, Seoul 06978, Korea
}

(Received 7 October 2015, Received in final form 11 November June 2015, Accepted 16 November 2015)

\begin{abstract}
In this paper, the safety distances and maximum permissible power (MPP) of resonant wireless power transfer systems are defined and derived with regard to human exposure to electromagnetic field (EMF). The definition is based on the calculated induced current density and electric field in the standard human model located between the transmitting and receiving coil. In order to avoid the adverse health effects such as stimulation of nerve tissues, the induced current and electric field must not exceed the basic restriction values specified in EMF safety guidelines. The different combinations of diameters of the coils and the distance between the two coils are investigated and their effects are analyzed. Two versions of EMF safety guidelines (ICNIRP 1998 and ICNIRP 2010) are used as bases for safety distance calculation and the difference between the two guidelines are discussed.
\end{abstract}

Keywords : human model, induced current, maximum permissible power, safety distance, wireless power transfer

\section{Introduction}

Wireless power transfer (WPT) technique utilizes inductive coupling, resonant inductive coupling, capacitive coupling, far field radiation, or other electromagnetic coupling to transfer electric power without wires. Resonant inductive coupling technique or simply, resonant coupling was first explored in the pioneering experiments of Nikola Tesla [1]. However, it was not until 2007 when Soljacic et al. [2] revisited the technology to transmit 60 $\mathrm{W}$ power over $2 \mathrm{~m}$ distance that the active research work started again on resonant coupling to increase the transmission distance while maintaining high efficiency. However, despite considerable amount of work [3-6], there are still very few commercial products that utilizes resonant coupling to transfer power over distances greater than a few centimeters.

One of the main difficulties associated with resonant WPT over a moderate distance is the electromagnetic field (EMF) safety. When the human body is located between receiving and transmitting coils, the time-varying magnetic field of the WPT system induces electric field

(C)The Korean Magnetics Society. All rights reserved.

*Corresponding author: Tel: +82-2-820-0644

Fax:+82-2-817-7961, e-mail: jkbyun@ssu.ac.kr and current inside the human body. According to the various EMF safety guidelines [7, 8], these induced current or electric field can lead to excitation of nerve tissues or other negative health effects, and they should be kept lower than exposure limits defined in the guidelines to avoid these negative effect.

Induced current and electric field inside the human body are affected by many factors, including the characteristics of the magnetic field source (transmitting coil), the distance of human body from the source, the magnitude of input power, and characteristics of the human body exposed to the magnetic field. Since it is difficult to measure actual induced current in the human body, numerical analysis is often used to calculate the induced current and determine if the limit has been exceeded [8]. In these numerical analyses, anatomical human body models with heterogeneous tissue composition or simplified homogeneous human models can be used. The homogeneous models tend to overestimate the induced current, but they are still widely used for conservative approach [9].

For a resonant WPT system, two parameters can be defined with regards to human EMF safety. Safety distance can be defined for a system with fixed maximum input power. It is the minimum distance from the transmitter or receiver coil of WPT to the human body that will keep 


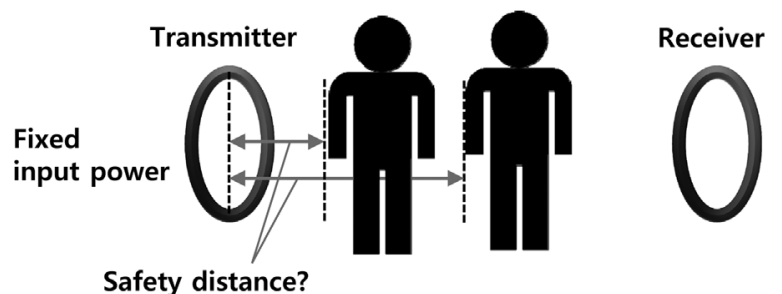

Safety distance?

(a)

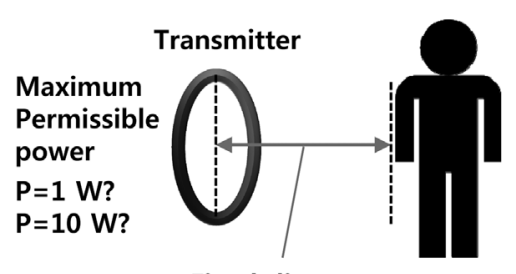

Fixed distance

(b)

Fig. 1. Concept of safety distance and maximum permissible power (MPP) of a WPT system. (a) Safety distance when maximum input power is fixed. (b) MPP when minimum distance is fixed.

the induced current or electric field in the human body lower than the limit. If human body is closer to the coil than the safety distance, the induced current may exceed the limit. Thus, the manufacturer of a WPT system should take necessary actions, such as making a protective case around the coil with sufficient inner space, to ensure that people will not get closer to the coil than the safety distance.

On the contrary, if a WPT system has a pre-determined minimum distance human can approach, for example, due to the dimension of the product or limits on installation space, its maximum permissible power (MPP) can be defined as the maximum input power that will keep the induced current lower than the limit when the human body is placed at that fixed distance. These concepts are explained in Fig. 1.

In this paper, safety distance and MPP are derived for various configurations of resonant WPT systems based on induced current density and electric field values in the human body model calculated by numerical analysis. The WPT systems have double loop coils and are designed to operate around $150 \mathrm{kHz}$, with varying coil diameters and distance between transmitter and receiver coils. For human body model, homogeneous body model specified in IEC-62311 standard was used. Two widely used EMF safety guidelines, ICNIRP (international commission on non-ionizing radiation protection) 1998 and ICNIRP 2010, are used as bases of the safety distance and MPP derivation, and differences between the two guidelines are discussed.

\section{Design of WPT Systems and Standard Human Model}

\subsection{Design of resonant WPT systems}

The resonant WPT system is designed with double loop structure shown in Fig. 2 [3]. It is composed of 4 coils, and the resonant frequency is set to be $150 \mathrm{kHz}$.

The inductance of the resonant coil $L$ can be calculated as follows [10],

$$
L=0.01595 D\left(2.303 \log _{10} \frac{8 D}{w}-2+\mu_{r} \delta\right),
$$

where $D$ is the diameter of the coil loop, $w$ is the diameter of coil wire, $\mu_{r}$ is the relative permeability of the wire (copper), and $\delta$ is the skin effect correction factor determined by resonant frequency $f$ and wire diameter $w$ as shown in Fig. 3. The resonant capacitor is connected in series with the resonant coil, and its capacitance $C$ can be

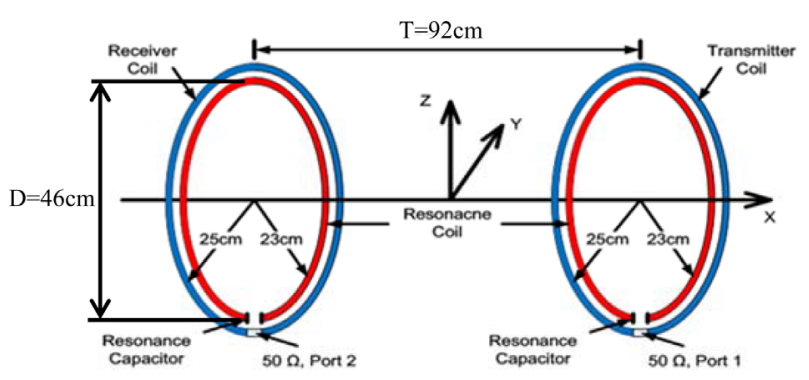

Fig. 2. (Color online) Schematics of the resonant WPT system with double loop $(D=46 \mathrm{~cm}, T=92 \mathrm{~cm})$ [3].

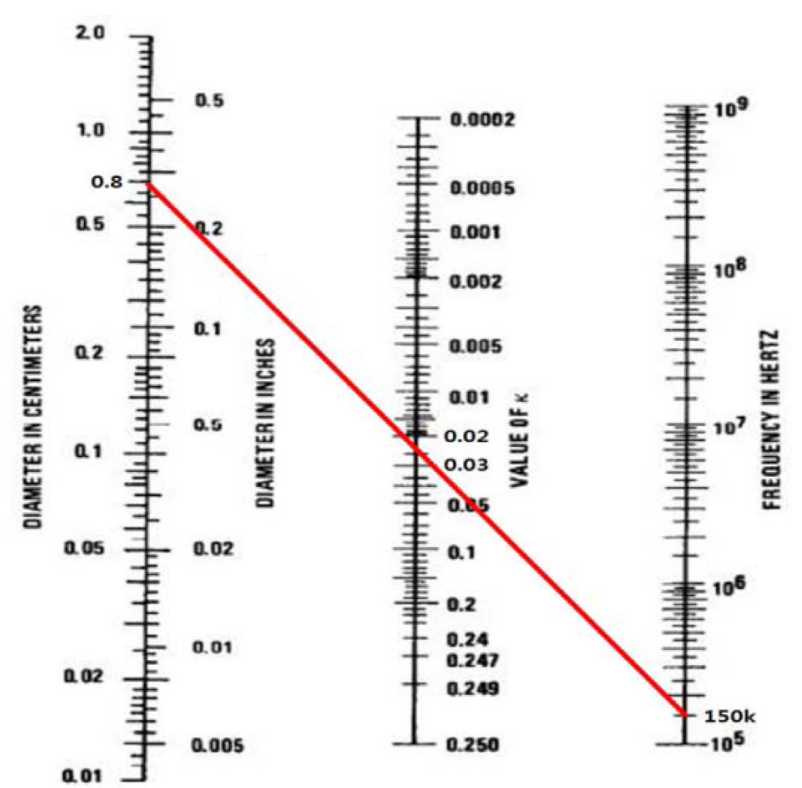

Fig. 3. (Color online) Skin effect correction factor $\delta$ according to wire diameter $w$ and frequency $f[10]$. 
Table 1. Design components of resonant WPT systems.

\begin{tabular}{lcccc}
\hline \multicolumn{1}{c}{ Design components } & System 1 & System 2 & System 3 & System 4 \\
\hline Resonant coil diameter $D[\mathrm{~cm}]$ & 46 & 46 & 92 & 92 \\
Transmission distance $T[\mathrm{~cm}]$ & 67 & 92 & 67 & 92 \\
Resonant frequency $[\mathrm{kHz}]$ & 150 & 150 & 150 & 150 \\
Port impedance $[\Omega]$ & 50 & 50 & 50 & 50 \\
Coil wire material & copper & copper & copper & copper \\
Wire diameter $[\mathrm{mm}]$ & 8 & 0.0256 & 0.0256 & 8 \\
Skin effect correction factor & 0.0256 & 1.20 & 2.80 & 0.0256 \\
Resonant coil inductance $[\mu \mathrm{H}]$ & 1.20 & 0.938 & 0.402 & 2.80 \\
Capacitance $[\mu \mathrm{F}]$ & 0.938 & & & 0.402 \\
\hline
\end{tabular}

calculated from,

$$
C=\frac{1}{(2 \pi f)^{2} L} .
$$

Four different WPT systems are designed with different combinations of coil loop diameter $D$ and transmission distance $T$ (distance between transmitter and receiver coils), and induced current distribution in the standard human model is calculated by numerical analysis for each system. The design components of four WPT systems are summarized in Table 1.

\subsection{Standard human model for induced current cal- culation}

Various human models can be used for numerical modeling purposes. 3D anatomical models are often constructed based on series of 2D computerized tomography (CT) or magnetic resonance (MR) images separated by short distance (usually less than $1 \mathrm{~mm}$ ). These are adequate for detailed dosimetry and precise analysis of induced current distribution in different biological tissues. However, they often require a lot of development time and cost. On the other hand, for the purpose of determining the compliance with EMF guidelines, a simplified body shape of uniform conductivity is often used. The dielectric properties of such a model are often the whole body average at the frequencies being investigated, but can be representative of particular body parts or tissue types instead. These models tend to overestimate the current density when in the near field [9].

In this paper, homogeneous body model specified in IEC-62311 standard was used for induced current and electric field calculation. Fig. 4 and 5 show the description of the model and details of the head and shoulders [9]. The model is composed of head and torso part, and the height of the model is about $150 \mathrm{~cm}$. The dielectric property of the muscle tissue is used for the whole body. The electrical conductivity was $\sigma=0.37265 \mathrm{~S} / \mathrm{m}$ and

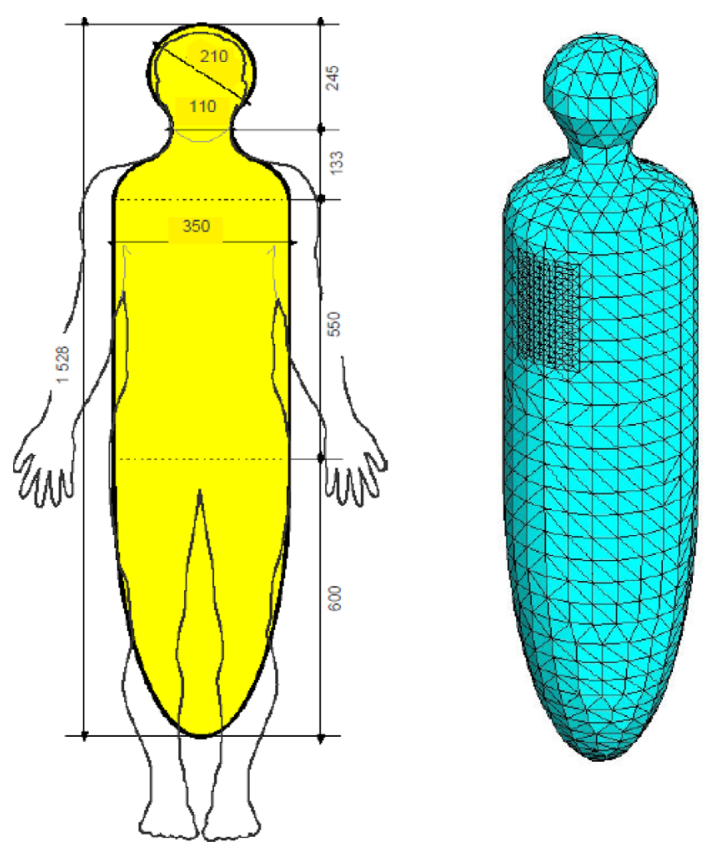

Fig. 4. (Color online) Full view of standard homogeneous human body model [9].

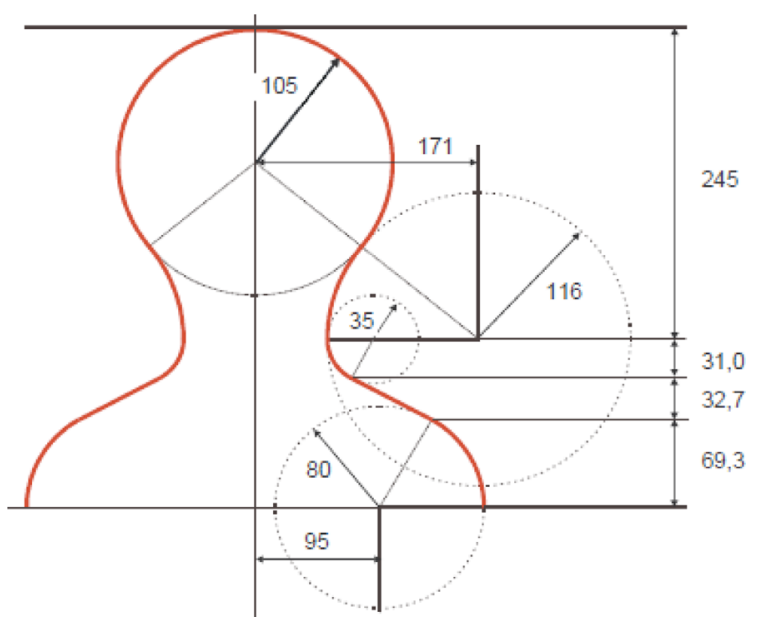

Fig. 5. (Color online) Detailed view of head and shoulder of standard body model [9]. 
relative permittivity was set as $\varepsilon_{r}=7109.1$ at $150 \mathrm{kHz}$ according to the works of Gabriel et al. [11].

\section{Calculation of Induced Current in Body Model}

In this chapter, the resonant WPT system and the standard human model introduced in Chapter 2 are used for the calculation of the induced current and electric field in the body model. For numerical analysis of the WPT systems, ANSYS HFSS software was used, which is based on finite element method. The vector wave equation for electric field $\mathbf{E}$ is used for the governing equation of the finite element method, which can be written as follows,

$$
\nabla \times\left(\frac{1}{\mu_{r}} \nabla \times \mathbf{E}\right)-k_{0}^{2} \varepsilon_{r} \mathbf{E}=-j k_{0} Z_{0} \mathbf{J},
$$

where $\mathbf{E}$ is the electric field, $\mathbf{J}$ is the current density, $\varepsilon_{r}$ and $\mu_{r}$ are the relative permittivity and relative permeability, respectively, $k_{0}$ is the wavenumber in free space, and $Z_{0}$ is the intrinsic impedance of free space.

Four WPT systems are considered with different coil

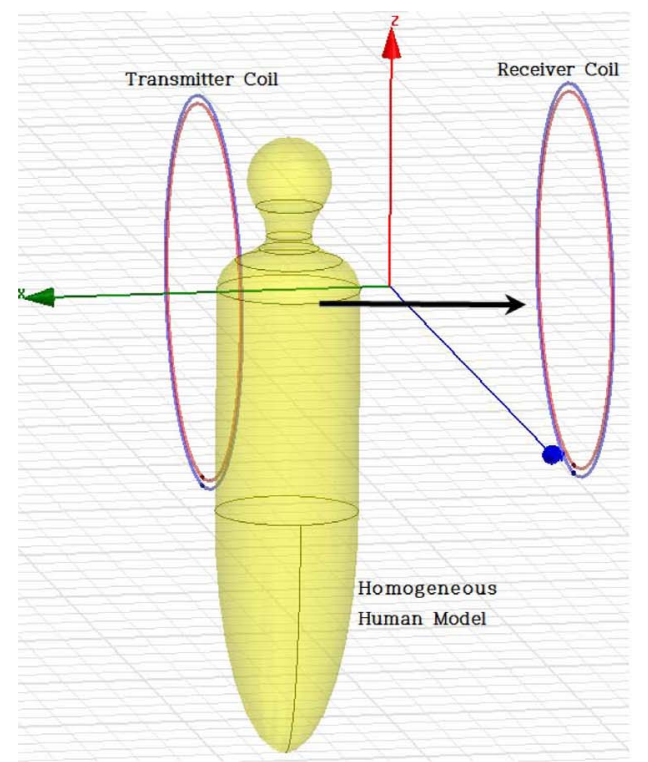

(a) diameter $D$ and transmission distance (distance between coils) $T$ as shown in Fig. 6 and Table 1. First, the efficiency of each system is calculated and compared without the standard human model. The transmission efficiency $\eta$ can be obtained from $S_{21}$ parameter. $S_{21}$ can be calculated from the following equation,

$$
S_{21}[\mathrm{~dB}]=10 \log _{10} \frac{P_{o}}{P_{i}}
$$

where $P_{i}$ is the input power and $P_{o}$ is the output power. Table 2 shows the efficiency $\eta$ and frequency of maximum efficiency (resonant frequency) for 4 WPT systems.

As can be expected, it can be seen from Table 2 that efficiency $\eta$ is increased as coil diameter $D$ is increased and transmission distance $T$ is decreased. Also, as $\eta$ is increased and $T$ becomes smaller compared to $D$ (system 3 ), the resonant frequency is slightly moved from the initial design value of $150 \mathrm{kHz}$ because the mutual inductance and capacitance are increased, which in turn affects the resonant frequency. This suggests that for a practical resonant WPT system, it is necessary to monitor the system efficiency and adjust the switching frequency of the input coil as the relative coil locations are changed

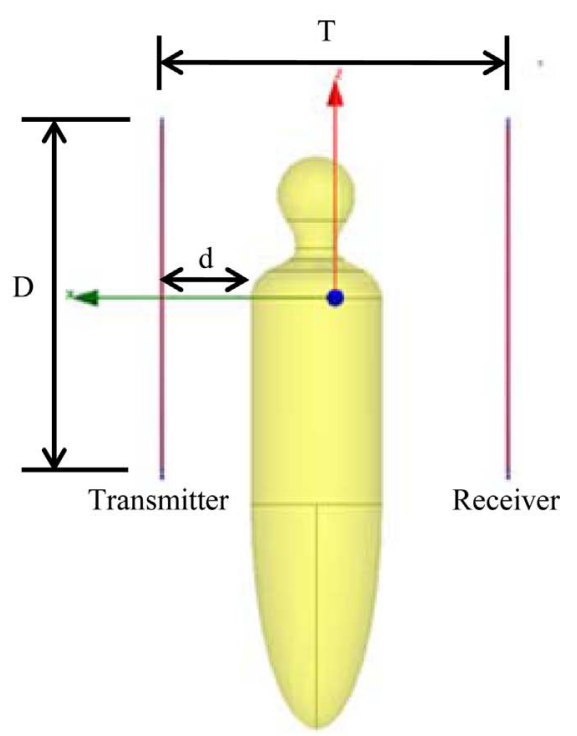

(b)

Fig. 6. (Color online) Human body model and resonant WPT system. (a) Change of location of body model between WPT coils. (b) Definition of distance $d$ from transmitter to body model.

Table 2. Maximum efficiency of WPT systems according to coil diameter $D$ and transmission distance $T$.

\begin{tabular}{lcccc}
\hline \multicolumn{1}{c}{ System no. } & System 1 & System 2 & System 3 & System 4 \\
& $(D=46 \mathrm{~cm}, T=67 \mathrm{~cm})$ & $(D=46 \mathrm{~cm}, T=92 \mathrm{~cm})$ & $(D=92 \mathrm{~cm}, T=67 \mathrm{~cm})$ & $(D=92 \mathrm{~cm}, T=92 \mathrm{~cm})$ \\
\hline Maximum efficiency $\eta[\%]$ & 35.09 & 11.47 & 72.40 & 66.76 \\
Frequency of maximum efficiency $[\mathrm{kHz}]$ & 150 & 150 & 148 & 150 \\
\hline
\end{tabular}


in order to maintain the maximum efficiency.

\subsection{Efficiency analysis according to the presence and location of the body model}

According to the previous works by the authors, the change in efficiency of resonant WPT system due to the presence of body model was less than $1 \%$ [12]. In this section, efficiency in the presence of human body is further analyzed according to the distance $d$ of the body model from the transmitting coil. Fig. 6 shows the standard human model placed between the coils of WPT system. The distance $d$ is defined from the center of the transmitting coil to the edge of human model as shown in Fig. 6. The minimum value of $d$ was set as $3.5 \mathrm{~cm}$. The efficiency change is analyzed as the human model is moved away from the transmitter coil. The distance increment for each calculation is $5 \mathrm{~cm}$.

Table 3 shows the efficiency analysis for system 4 $(D=96 \mathrm{~cm}$ and $T=96 \mathrm{~cm})$. Although there is slight decrease in efficiency when the body approaches the transmitter or receiver, the change in efficiency is less than $0.18 \%$, and the resonant frequency is also unchanged at $150 \mathrm{kHz}$. Other systems (systems 1-3) showed similar results. Thus, it can be concluded that the effect of presence and location of human body on the efficiency of a WPT system is negligible.

\subsection{Induced current in human model according to the distance from transmitter}

In this section, induced current density in human body model is analyzed according to the distance $d$ between body model and the transmitter. Reference input power of transmitter is set as $1 \mathrm{~W}$ in this section. First, before the induced current density is calculated, the magnetic field pattern of a WPT system without the human body is analyzed by extracting the magnetic field data within a cuboid shaped air box $\left(92 \times 92 \times 5 \mathrm{~cm}^{3}\right)$ as shown in Fig. 7. The maximum, averaged, and 99th-percentile value of magnetic field in cuboid is plotted in Fig. 8 for system 4 $(D=92 \mathrm{~cm}$ and $T=92 \mathrm{~cm})$ as the distance of the cuboid from the transmitter is changed. The magnetic field intensity is highest near the transmitter, reaches minimum value near the midpoint slanted to the receiver, and increases again near the receiver. The ratio of the highest field value to the lowest one is about 5.07 for 99th-

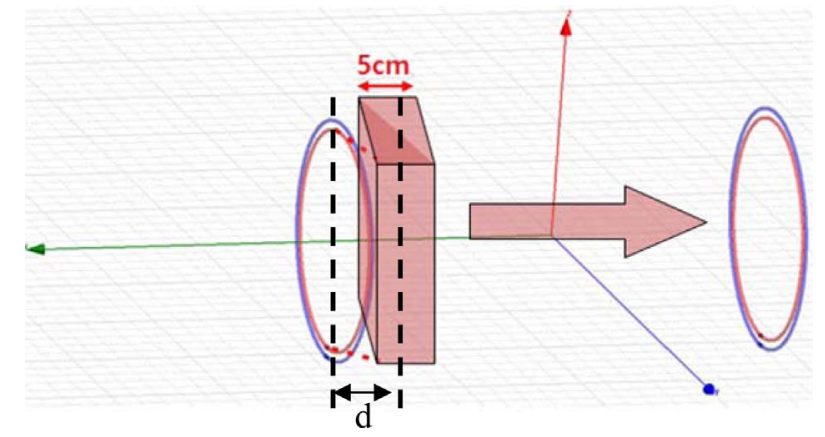

Fig. 7. (Color online) Cuboid shaped air-box for magnetic field calculation.

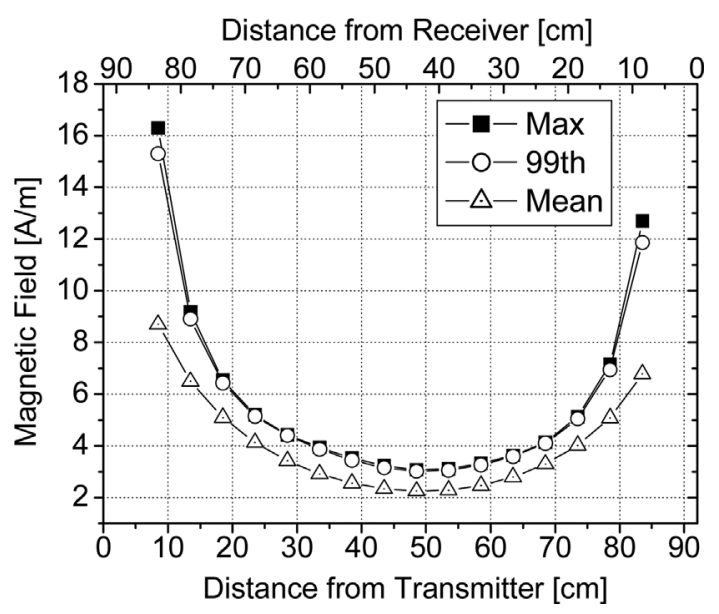

Fig. 8. Magnetic field pattern of WPT system $4(D=92 \mathrm{~cm}$, $T=92 \mathrm{~cm}$ ) without human model (each point is calculated in a cuboid of $92 \times 92 \times 5 \mathrm{~cm}^{3}$ ).

percentile values. The inhomogeneous field pattern, in turn, is expected to cause similar variation in calculated induced current density as location of the body model is changed.

It should be noted that the maximum value of magnetic field or induced current density in certain volume can be affected by various factors such as size and quality of meshes and the type of numerical method or software used. Thus, it is recommended by EMF guidelines that 99th-percentile values should be used instead of maximum values when numerical analysis is used to determine compliance with exposure limits $[8,13]$. Hence, 99thpercentile value of induced current density and electric field inside the human body model is used to derive the maximum permissible power and safety distance in this

Table 3. Maximum efficiency according to distance $d$ of human model from the transmitter $(D=92 \mathrm{~cm}, T=92 \mathrm{~cm})$.

\begin{tabular}{lccccccccccc}
\hline \hline Distance from transmitter $d[\mathrm{~cm}]$ & 3.5 & 8.5 & 13.5 & 18.5 & 23.5 & 28.5 & 33.5 & 38.5 & 43.5 & 48.5 & 53.5 \\
\hline Efficiency $\eta[\%]$ & 66.53 & 66.51 & 66.64 & 66.62 & 66.65 & 66.65 & 66.70 & 66.58 & 66.58 & 66.55 & 66.55 \\
Resonant frequency $[\mathrm{kHz}]$ & 150 & 150 & 150 & 150 & 150 & 150 & 150 & 150 & 150 & 150 & 150 \\
\hline
\end{tabular}




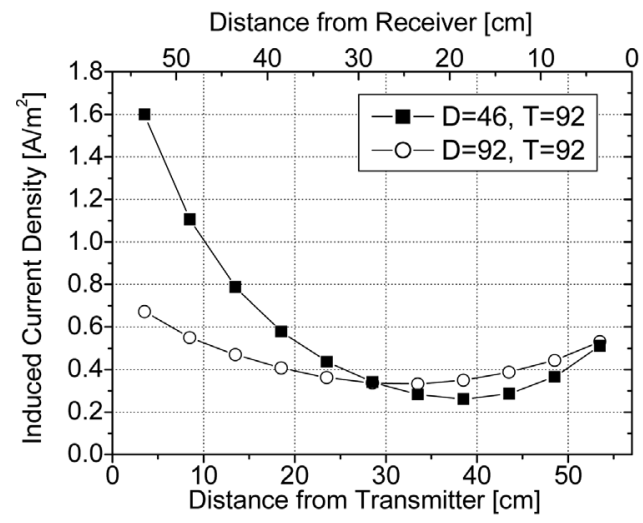

(a)

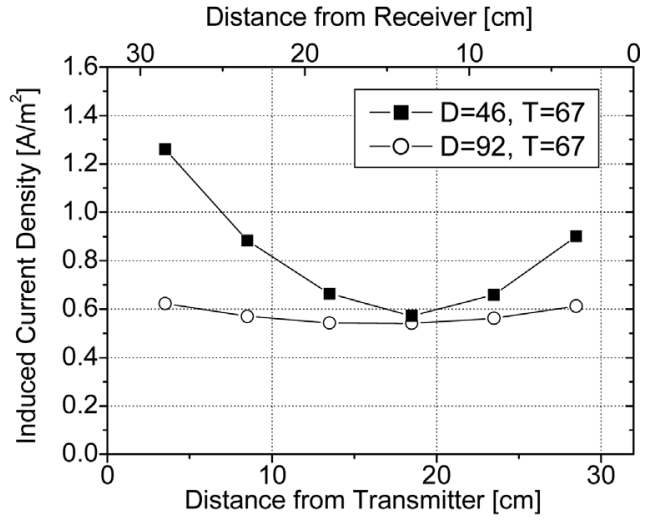

(b)

Fig. 9. 99th-percentile values of induced current density in human model according to the distance of the body model from the transmitter. (a) System 1 and 3 (transmission distance $T=67 \mathrm{~cm}$ ). (b) System 2 and 4 (transmission distance $T=92 \mathrm{~cm}$ ).

paper.

Fig. 9 shows the 99th-percentile values of induced current density in the standard human model as the human model is moved from near the transmitter to the receiver. The overall pattern is similar to the magnetic field pattern shown in Fig. 8, and WPT systems with smaller coil diameter $(D=46 \mathrm{~cm})$ show larger variation of induced current density as the body location is changed. System 2 $(D=46 \mathrm{~cm}, T=92 \mathrm{~cm})$ shows the largest variation, with the highest induced current density $\left(1.60 \mathrm{~A} / \mathrm{m}^{2}\right)$ at $3.5 \mathrm{~cm}$ from the transmitter and lowest value $\left(0.26 \mathrm{~A} / \mathrm{m}^{2}\right)$ at 38.5 $\mathrm{cm}$ from the transmitter.

\section{Calculation of MPP and Safety Distance}

\subsection{EMF safety guidelines}

In this chapter, the induced current density in human model calculated in the previous chapter is used to derive the maximum permissible power (MPP) and safety distance of the WPT systems based on EMF safety guidelines. Two kinds of international EMF safety guidelines are considered in this paper, i.e., ICNIRP 1998 and ICNIRP 2010 guidelines. ICNIRP guidelines are endorsed by world health organization (WHO). It was first published in 1998 , but the low-frequency $(1 \mathrm{~Hz}$ to $100 \mathrm{kHz})$ guideline was revised in 2010 taking into account recent advances in the scientific knowledge [7,8]. Different countries have adopted different EMF guidelines depending on many factors. For example, Germany and Japan have adopted ICNIRP 2010 guideline, while many other EU countries still adopt ICNIRP 1998 guideline.

The exposure limits (basic restrictions) of the two guidelines are summarized in Table 4 and 5 for $150 \mathrm{kHz}$, which is the resonant frequency of the WPT system. The occupationally exposed population consists of adults who are trained to be aware of potential risk and to take appropriate precautions. By contrast, the general public comprises individuals of all ages and of varying health status. In many cases, members of the general public are unaware of their exposure to EMF [7]. For both guidelines, basic restrictions are defined that are based on established health effects. These basic restrictions must not be exceeded in order to avoid adverse health effects. In the ICNIRP 1998, induced current density $J$ was adopted as physical quantity of basic restriction, whereas internal electric field $E$ was adopted in ICNIRP 2010. $J$ and $E$ are related by the following equation,

$$
J=\sigma E,
$$

where $\sigma$ is the conductivity of the tissue. The change of basic restriction in ICNIRP guideline results in more lenient exposure limits as will be discussed a later section.

Table 4. Basic restrictions (exposure limits) of the ICNIRP 1998 guideline at $150 \mathrm{kHz}$ [7].

\begin{tabular}{cc}
\hline \hline $\begin{array}{c}\text { Exposure } \\
\text { characteristic }\end{array}$ & $\begin{array}{c}\text { Current density for head and trunk }\left[\mathrm{A} / \mathrm{m}^{2}\right] \\
(\mathrm{rms})\end{array}$ \\
\hline Occupational & 1.5 \\
General public & 0.3 \\
\hline
\end{tabular}

Table 5. Basic restrictions (exposure limits) of the ICNIRP 2010 guideline at $150 \mathrm{kHz}$ [8].

\begin{tabular}{cc}
\hline \hline Exposure characteristic & Internal electric field [V/m] (rms) \\
\hline Occupational & 40.5 \\
General public & 20.25 \\
\hline
\end{tabular}




\subsection{Calculation of MPP and safety distance based on basic restrictions}

In the previous chapter, the 99th-percentile values of induced current density $\left(J_{r e f}\right)$ and electric field $\left(E_{r e f}\right)$ in the human model were calculated for the reference input power of $P_{r e f}=1 \mathrm{~W}$. Since the electric conductivity of human body does not change much with respect to the varying magnetic field [11], it can be assumed that WPT systems with the body model are linear, in which case the changes in induced current density and electric field are proportional to the square root of change in input power. These relations are shown in the following equations:

$$
\begin{aligned}
& J^{\prime}=\sqrt{\frac{P^{\prime}}{P_{r e f}}} J_{r e f}, \\
& E^{\prime}=\sqrt{\frac{P^{\prime}}{P_{r e f}}} E_{r e f},
\end{aligned}
$$

where $J^{\prime}$ and $E^{\prime}$ are the induced current density and electric field for the specific location of the body model when input power is $P^{\prime}$, and $J_{\text {ref }}$ and $E_{\text {ref }}$ are the calculated current density and electric field for the same location of the body model when reference input power is $P_{\text {ref. }}$. From (6) and (7), we can derive the equations for the input power required to obtain specific current density $J^{\prime}$ or electric field $E^{\prime}$ in the body model as follows,

$$
\begin{aligned}
& P^{\prime}=\left(\frac{J^{\prime}}{J_{r e f}}\right)^{2} P_{r e f}, \\
& P^{\prime}=\left(\frac{E^{\prime}}{E_{r e f}}\right)^{2} P_{r e f} .
\end{aligned}
$$

(8) and (9) can be directly used to calculate the MPP of the WPT systems. MPP based on the ICNIRP 1998 can be obtained from (8) with $J^{\prime}=0.3 \mathrm{~A} / \mathrm{m}^{2}$, which is the basic restriction of that guideline for the general public at $150 \mathrm{kHz}$. MPP based on the ICNIRP 2010 can be obtained from (9) with $E^{\prime}=20.25 \mathrm{~V} / \mathrm{m}$, which is the basic restriction of that guideline for the general public.

Fig. 10 shows the MPP for the 4 WPT systems accord-

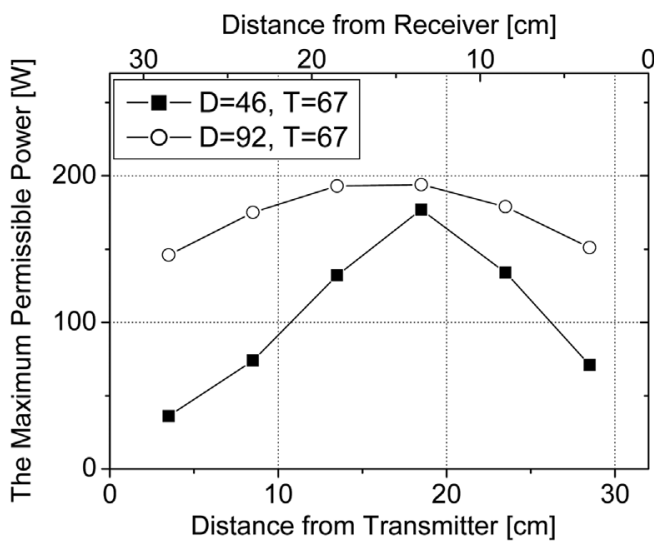

(a)
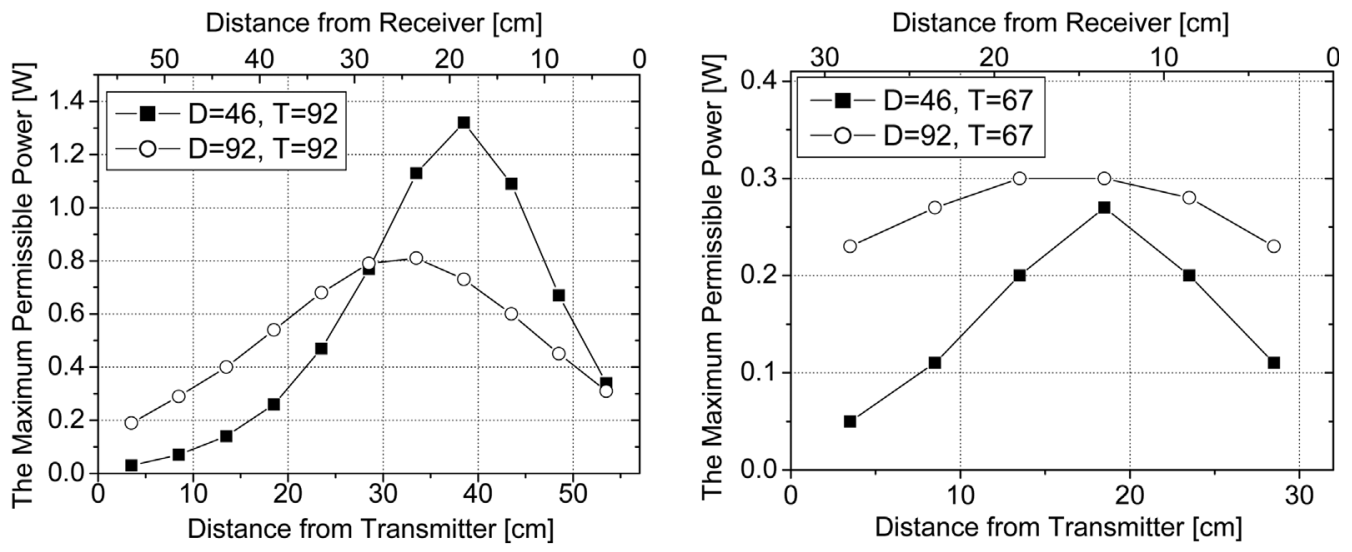

(b)

Fig. 10. MPP plots according to the distance of body model from the coils. (a) MPP plots based on ICNIRP 2010 guidelines. (b) MPP plots based on ICNIRP 1998 guidelines. 
ing to the location of the body model from the transmitter. Fig. 10(a) shows MPP plots based on ICNIRP 2010 whereas Fig. 10(b) shows those based on ICNIRP 1998. It can be seen that maximum MPP in Fig. 10 appears at the same distance where minimum $J_{\text {ref }}$ was obtained in Fig. 9 (midpoint slanted to receiver), which can be explained by (8). Also, MPP is decreased as the body model approaches transmitter or receiver, and the minimum MPP is obtained near the transmitter as expected.

Comparing the two guidelines, the MPP of ICNIRP 2010 is about 640 times greater than that of ICNIRP 1998. This is due to the difference of basic restriction values shown in Table 4 and 5. Using (5) and the conductivity of the homogeneous human body model, the corresponding current density of ICNIRP 2010 is calculated as $J_{2010}^{\prime}=\sigma E^{\prime}=0.37265 \times 20.25 \cong 7.546 \mathrm{~A} / \mathrm{m}^{2}$, which is about 25 times greater than $J_{1998}^{\prime}=0.3 \mathrm{~A} / \mathrm{m}^{2}$. From (8), the MPP is proportional to the square of $J^{\prime}$, which results in the great difference shown in Fig. 10(a) and (b).

This does not mean that basic restriction of ICNIRP 2010 guideline is precisely 25 times more lenient than that of ICNIRP 1998, since actual human body is composed of inhomogeneous tissues with varying conductivity values. Rather, it is a direct result of dosimetry based on body model with uniform conductivity value. However, it is safe to say that magnetic field exposure limits of ICNIRP 2010 are more lenient than those of ICNIRP 1998 in general. For example, the MPP of system $4(D=$ $92 \mathrm{~cm}, T=92 \mathrm{~cm}$ ) at $8.5 \mathrm{~cm}$ from the transmitter is 192 W for ICNIRP 2010, but only 0.3 W for ICNIRP 1998. Consequently, only limited low to mid-power applications are feasible based on ICNIRP 1998, but high power applications are possible based on ICNIRP 2010.

It should be noted that the calculations made in this paper is very conservative (high value of calculated induced current density) because of several factors. First, homogeneous human model tends to overestimate the induced current density compared to anatomical human models [9]. Second, the conductivity of homogeneous human model was taken as that of muscle $(\sigma=0.37265 \mathrm{~S} /$ m) for MPP calculations. However, volume averaged conductivity of all tissues in an anatomical human model (male adult) is about $0.24 \mathrm{~S} / \mathrm{m}$ [14]. Thus, the actual induced current should be a little lower and the actual MPP should be a little higher. Nevertheless, even when these factors are taken into consideration, MPP of system 4 at $8.5 \mathrm{~cm}$ from the transmitter should still be less than 1 W based on ICNIRP 1998.

In terms of coil diameter $D$, larger diameter resulted in higher MPP in general except for a few points near the receiver when $T=92 \mathrm{~cm}$. This is due to the fact that

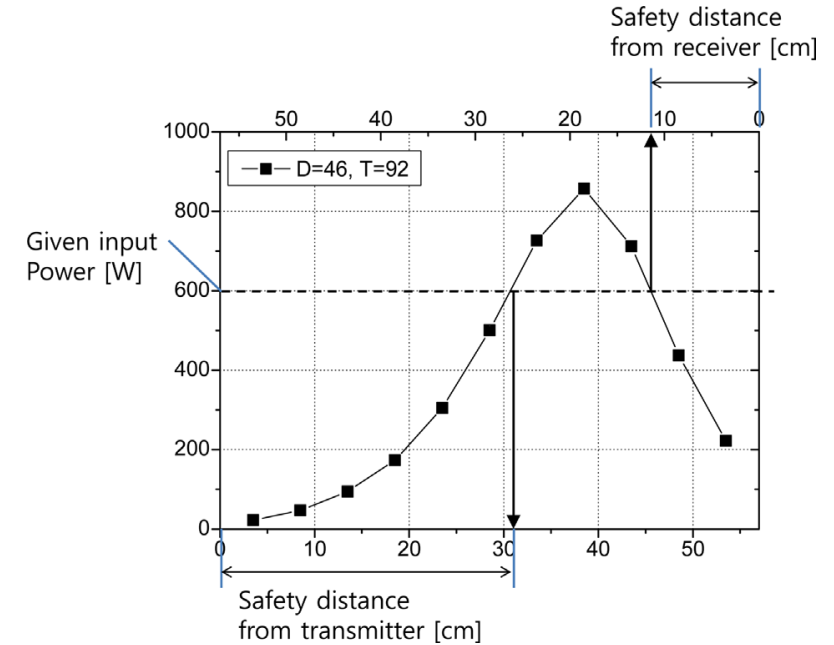

Fig. 11. (Color online) Procedure to obtain safety distance for given input power from MPP plot.

smaller $D$ results in highly localized induced current distribution in body model, whereas larger $D$ results in more uniform distribution and lower 99th-percentile value of induced current density. The reason smaller $D$ shows higher MPP at a few points in Fig. 10 is that the magnetic field near the receiver is very low for system $2(D=46$ $\mathrm{cm}, T=92 \mathrm{~cm}$ ) due to its low efficiency.

The safety distance of the WPT systems can be directly obtained from Fig. 10. For each curve in Fig 10, horizontal line with given input power will determine two intersections with the curve. $x$-coordinates of those intersections are safety distance from the transmitter and that from the receiver, respectively. This procedure is explained in Fig. 11 for given input power of $600 \mathrm{~W}$. Fig. 12 shows the safety distance plots for the WPT systems. All plots show monotonous increase as the input power is increased. Also, the safety distance from the receiver is shorter than that from the transmitter in all cases, since the magnetic field is lower near the receiver. Safety distances based on ICNIRP 2010 are much smaller than those based on ICNIRP 1998. For example, safety distance from the transmitter of system $2(D=46 \mathrm{~cm}, T=92 \mathrm{~cm})$ for input power of $1 \mathrm{~W}$ is obtained as about $0.16 \mathrm{~cm}$ for ICNIRP 2010, which means virtually the entire space between the coils is safe. But it becomes $32 \mathrm{~cm}$ for ICNIRP 1998. This again confirms that recently revised ICNIRP 2010 guideline is much more lenient compared to ICNIRP 1998.

\section{Conclusions}

In this paper, MPP and safety distance of resonant WPT systems were derived from the induced current density 

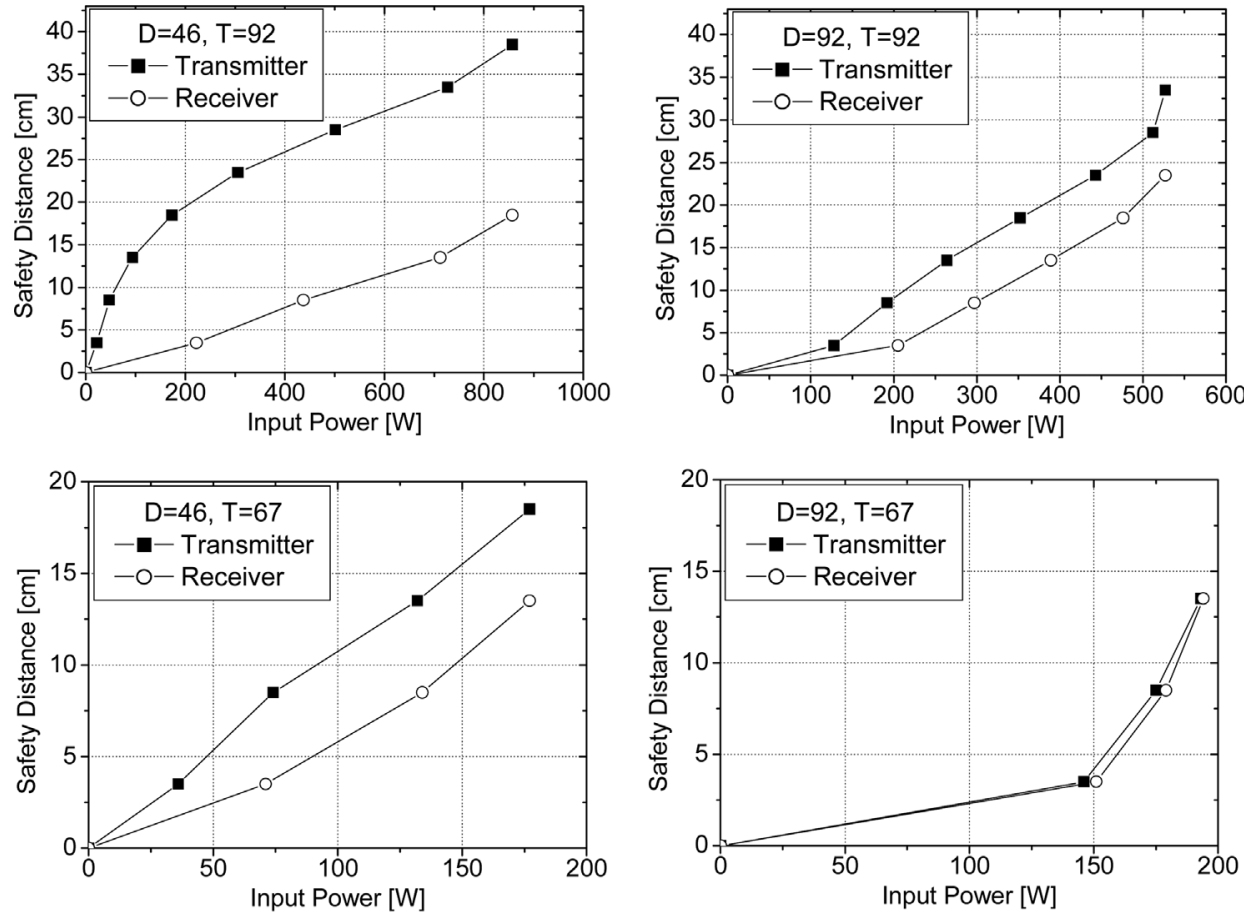

(a)
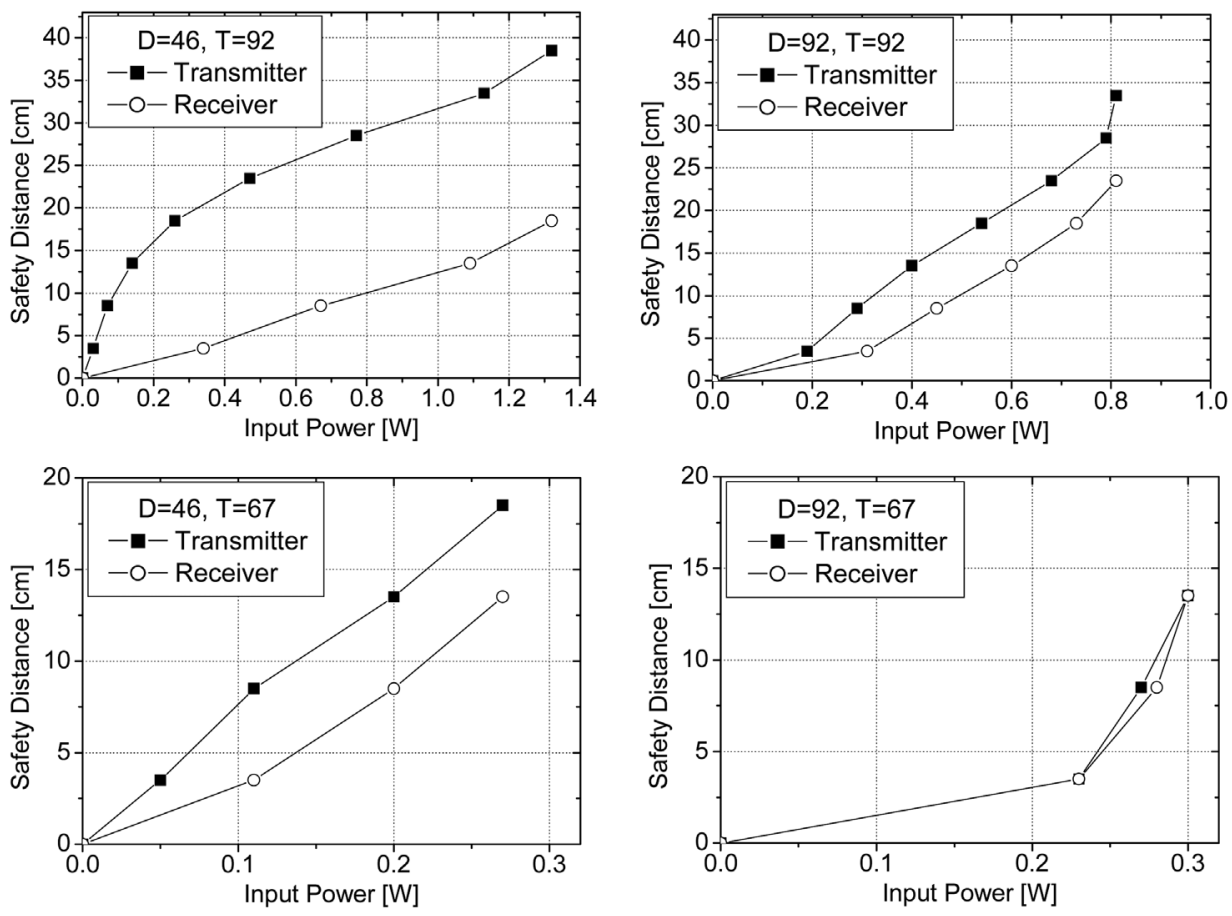

(b)

Fig. 12. Safety distance plots according to the varying input power. (a) Safety distance plots based on ICNIRP 2010 guidelines. (b) Safety distance plots based on ICNIRP 1998 guidelines.

and electric field calculation in a standard homogeneous body model. The derivations are based on exposure limit values outlined in ICNIRP 1998 and ICNIRP 2010 EMF safety guidelines. WPT systems with larger coil diameter and higher efficiency exhibited higher MPP and lower safety distance, which means they are generally safer in terms of human exposure to magnetic field. This is because larger coil diameter leads to more uniform magnetic field 
and induced current distribution, whereas smaller coil diameter results in highly localized induced current distribution in body model, leading to higher 99th-percentile value. Comparing ICNIRP 1998 and ICNIRP 2010, ICNIRP 2010 was shown to have much more lenient and forgiving exposure limits based on dosimetry using homogeneous body model. Thus, high power resonant WPT systems are possible based on ICNIRP 2010, whereas only limited low to mid-power applications are feasible based on ICNIRP 1998. Since ICNIRP 2010 claims it is based on recent advances in the scientific knowledge [8], it may be reasonable to think that most countries should adopt that guideline. However, only a few countries have adopted ICNIRP 2010 in reality, because Governments need to look at many factors before deciding whether and how to implement the guidelines. Thus, continuing efforts need to be made to improve efficiency of WPT systems and obtain higher MPP and lower safety distance based on ICNIRP 1998 guidelines. It is expected that the procedures proposed in this paper can be applied to derive MPP and safety distance of various resonant WPT systems, and determine their compliance with EMF guidelines.

\section{Acknowledgements}

This work was supported by the Human Resources Development program (No. 20144030200600) of the Korea Institute of Energy Technology Evaluation and Planning (KETEP) grant funded by the Korea government Ministry of Trade, Industry and Energy.

\section{References}

[1] D. H. Childress, The Tesla Papers, Adventures Unlimited Press, Illinois (2000).

[2] A. Kurs, A. Karalis, R. Moffatt, J. D. Joannopoulos, P. Fisher, and M. Soljačić, Science 317, 83 (2007).

[3] A. Karalis, J. D. Joannopoulos, and M. Soljačić, Annals of Physics 323, 34 (2008).

[4] A. P. Sample, D. A. Meyer, and J. R. Smith, IEEE Trans. Ind. Electro. 58, 544 (2011).

[5] Study on Arrangement of Self-Resonant Coils in Wireless Power Transfer System Based on Magnetic Resonance, The Journal of Korea Electromagnetic Engineering Society 21, 564 (2010).

[6] S. Y. R. Hui, W. Zhong, and C. K. Lee, IEEE Trans. Power Electr. 29, 4500 (2014).

[7] International Commission on Non-Ionizing Radiation Protection, Health Physics 74, 494 (1998).

[8] International Commission on Non-Ionizing Radiation Protection, Health Physics 99, 818 (2010).

[9] International Standard IEC 62311, IEC, Geneva (2007).

[10] F. E. Terman, Radio Engineers Handbooks, First Edition, McGraw-Hill, London (1950).

[11] S. Gabriel, R. W. Lau, and C. Gabriel, Phys. Med. Biol. 41, 2271 (1996).

[12] H. Shin and J.-K. Byun, Journal of the Korean Institute of Illuminating and Electrical Installation Engineers 27, 96 (2013).

[13] H.-J. Song, H. Shin, H.-B. Lee, J.-H. Yoon, and J.-K. Byun, IEEE Trans. Magn. 50, 7025804 (2014).

[14] A. Christ, W. Kainz, E. G. Hahn, K. Honegger, M. Zefferer, E. Neufeld, W. Rascher, R. Janka, W. Bautz, J. Chen, B. Kiefer, P. Schmitt, H.-P. Hollenbach, J. Shen, M. Oberle, D. Szczerba, A. Kam, J. W Guag, and N. Kuster, Phys. Med. Biol. 55, N23 (2010). 